\title{
Perspectiva do Gestor frente ao Alcoolismo no Contexto Laboral da Construção Civil
}

\author{
Jaqueline Garcia Fisch ${ }^{1}$, Tainara Kaspary ${ }^{2}$, \\ Cintia Sonale Rebonatto ${ }^{3}$, Carlos Costa ${ }^{4}$
}

\begin{abstract}
http://orcid.org/0000-0002-1851-4603 / IMED, Brasil
${ }^{2}$ http://orcid.org/0000-0002-6035-4279 / IMED, Brasil

${ }^{3}$ http://orcid.org/0000-0001-8638-0638 / IMED, Brasil

${ }^{4}$ http://orcid.org/0000-0003-0402-3763 / IMED, Brasil
\end{abstract}

O uso de bebidas alcoólicas é um problema de saúde pública que afeta, sobremaneira, a permanência e o desempenho de indivíduos no campo profissional. Diante dessa realidade, este trabalho objetivou analisar como os gestores atuam diante de contextos organizacionais em que há colaboradores alcoolistas. Para tanto se estudou, especificamente, os profissionais da construção civil no município de Passo Fundo, Rio Grande do Sul, Brasil. Metodologicamente realizou-se um estudo descritivo, de abordagem quantitativa e de corte transversal, aplicando-se questionários a 50 gestores de empresas do ramo. Os resultados demonstraram que, na perspectiva dos gestores, o consumo abusivo de álcool é uma doença e os casos nesse contexto laboral demonstraram a necessidade de ações internas destinadas ao uso consciente de bebidas alcoólicas. Portanto, os participantes da pesquisa consideraram o espaço laboral apropriado para a promoção de práticas preventivas; que, no entanto, acabam não sendo efetivadas.

Palavras-chave: álcool, trabalho, recursos humanos, construção civil, consumo de álcool.

\section{Managerial Perspectives on Alcoholism Amongst Construction Workers}

\section{Perspectiva del Gestor sobre el Alcoholismo en el Contexto Laboral de la Construcción Civil}

\section{Resumen}

As alcoholic beverage consumption represents an important public health issue, and is strongly tied to poor performance and permanence of individuals in professional fields, the present study's authors sought to analyse how managers act in the face of organizational contexts in which alcoholic employees are present. Professionals in civil construction from the municipality of Passo Fundo, State of Rio Grande do Sul, Brazil, were specifically targeted for study. Through a quantitative and crosssectional approach, a descriptive study was carried out by applying questionnaires to 50 managers of companies in the industry. From the managers' perspective, alcohol abuse is a disease. Cases in this work context demonstrate the need for internal actions aimed at limiting the conscious use of alcoholic beverages. While respondents considered the workplace to be an appropriate place to promote preventative measures against alcoholism, this was largely not carried out in practice.

Keywords: alcohol, work, human resources, civil construction, alcoholic consumption.
El consumo de bebidas alcohólicas es un problema de salud pública que afecta, fuertemente, el desempeño y permanencia de las personas en el campo profesional. Frente a esta realidad, este estudio tuvo como objetivo analizar cómo actúan los gestores en contextos organizacionales en los que hay empleados alcohólicos. Para ello, se estudiaron, específicamente, los profesionales de la construcción civil en el municipio de Passo Fundo, Rio Grande do Sul, Brasil. Metodológicamente, se realizó un estudio descriptivo, con un enfoque cuantitativo y de corte transversal, aplicando cuestionarios a 50 gestores de empresas del ramo. Los resultados mostraron que, desde la perspectiva de los gestores, el consumo abusivo de alcohol es una enfermedad y los casos en este contexto laboral evidencian la necesidad de acciones internas orientadas al uso consciente de bebidas alcohólicas. Por tanto, los participantes de la investigación consideraron el espacio de trabajo adecuado para la promoción de prácticas preventivas; que, sin embargo, acaban no llevándose a cabo.

Palabras clave: alcohol, trabajo, recursos humanos, construcción civil, consumo de alcohol. Trabalho, 21(3), 1560-1567. https://doi.org/10.5935/rpot/2021.3.20321 
O consumo habitual de álcool, definido como alcoolismo, gera problemas de saúde e, por conseguinte, a diminuição da qualidade de vida dos indivíduos (Martín-González et al., 2020). Nesse sentido, para além do aspecto individual, o consumo excessivo de bebidas alcoólicas influencia as dimensões políticas, econômicas, familiares e sociais, sobremaneira, nesse último viés, as relações de trabalho (Mendonça, Jesus, \& Lima, 2018). Assim sendo, o uso de bebidas alcoólicas é um problema de saúde pública e decorre do fato de os indivíduos experimentarem sensações agradáveis, apesar dos efeitos deletérios que a substância oferece (Calvo-Porral \& Levy-Mangin, 2019).

A Organização Mundial da Saúde (OMS) prevê um aumento significativo do consumo de álcool até 2025 no Brasil, tanto em relação à regularidade semanal quanto à quantidade de doses consumidas (WHO, 2018). A prevalência do abuso do álcool dentre os homens chega a $3,25 \%$, enquanto para mulheres $0,62 \%$. No que tange à dependência alcoólica, a prevalência dentre os homens é de $10,48 \%$ e para o sexo feminino é de 3,63\% (Laranjeira et al., 2012). Com base nesse cenário, reforça-se a preocupação quanto à dependência do uso do álcool em trabalhadores do sexo masculino, normalmente mais atuantes nas atividades braçais, como, por exemplo, na construção civil (Casarin, Lavarda, \& Silveira, 2018; Gavioli, Mathias, Rossi, \& Oliveira, 2014).

Em se tratando das consequências do alcoolismo no desempenho organizacional, verifica-se que impactam no absenteísmo, afastamentos, prejuízo das condições laborais, desligamentos e acidentes de trabalho (Gavioli et al., 2014; Gómez-Recasens, Alfaro-Barrio, Tarro, Llauradó, \& Solà, 2018). Ainda, estudos demonstraram que episódios frequentes de alcoolismo estão associados a distúrbios depressivos do trabalhador (Virtanen et al., 2018). Também, que o consumo de álcool reduz significativamente a qualidade das atividades básicas e avançadas realizadas na construção civil (Dzeng, Wang, \& Fang, 2015). Assim, quando o alcoolismo está relacionado aos trabalhadores desse setor, há uma preocupação relevante por essa ser considerada, normalmente, como uma atividade de alto risco de acidentes de trabalho.

Ante ao exposto, a abordagem dessa temática é prolífera em diversos estudos que relacionam o alcoolismo com o ambiente de trabalho e referem a preocupação com o abuso do álcool e os padrões nocivos de consumo (Brites, Abreu, \& Portela, 2019). No que se refere às particularidades dos contextos laborais, o tema foi discutido na perspectiva de trabalhadores do setor de serviços (Bravo Ortiz \& Marziale, 2010; Mabuchi, Oliveira, Lima, Conceição, \& Fernandes, 2007), da segurança pública (Lima, Dimenstein, Figueiró, Leite, \& Dantas, 2019), de transportes (Nascimento, Nascimento, \& Silva, 2007; Soares et al., 2007) e da área da saúde (Junqueira et al., 2017). Contudo, a percepção dos gestores frente ao problema tem sido pouco abordada.

Alguns estudos também foram realizados abordando o ramo da construção civil. Dentre esses, Casarin et al. (2018), Cockell (2008), Dzeng et al. (2015) e Lette, Ambelu, Getahun, \& Mekonen (2018) evidenciaram o consumo elevado de drogas lícitas e ilícitas entre os trabalhadores, em especial o álcool, agravado pela situação de vulnerabilidade social desses. Ainda, Bavafa, Mahdiyar e Marsono (2018) observaram a relação desse hábito com as questões de segurança, considerada uma das principais preocupações do ramo. Contudo, as pesquisas nesse campo são limitadas, necessitando de uma abordagem do tema sob a perspectiva dos gestores.

O ambiente laboral é um importante contexto social na vida do indivíduo, inclusive para aquele dependente de álcool (Castro, 2009) que pode estar travando lutas solitárias para a superação do problema (Fernandes, Santos, Santana, Teles, \& Silva, 2015). Assim, esses sujeitos necessitam de atenção especial por parte da organização e de seus gestores. Tais fatos, associados à necessidade de estudos contextualizados da construção civil na visão de seus gestores sobre o alcoolismo, justificam a relevância social e acadêmica do presente estudo. Acredita-se, nesse sentido, que por meio da prevenção do alcoolismo, contemplada por uma estrutura de saúde e segurança ocupacional, os problemas decorrentes dele no contexto laboral possam ser minimizados, inclusive no ramo da construção civil. Tal perspectiva ancora-se em estudo que já demonstrou a atitude positiva dos trabalhadores desse setor em relação à prevenção ao uso de álcool (Cheng \& Cheng, 2016). Assim, tem-se por hipótese que ações sistematizadas e contundentes de prevenção e enfrentamento ao alcoolismo possam reduzir sensivelmente os acidentes de trabalho. Ainda, contribuir com menores taxas de absenteísmo e, consequentemente, aumentar a produtividade do trabalhador e melhorar sua qualidade de vida profissional e pessoal, trazendo resultados positivos às organizações.

Dessa forma, realizou-se o presente estudo com o objetivo de analisar de que maneira os gestores atuam diante de contextos organizacionais em que há colaboradores alcoolistas. Para tanto se estudou, especificamente, os profissionais da construção civil no município de Passo Fundo, Rio Grande do Sul (RS), Brasil. Metodologicamente procedeu-se um estudo descritivo, de abordagem quantitativa e de corte transversal, aplicando-se questionários a 50 gestores de empresas do ramo, tendo-se em vista que esse setor apresenta importante participação no número de empresas do referido munícipio (Sebrae, 2020).

\section{Referencial Teórico}

Aborda-se nesta seção, primeiramente, as consequências negativas do uso do álcool, trazendo-se especificidades do contexto brasileiro. Após, parte-se por discorrer sobre o uso de álcool no contexto laboral, interesse precípuo dessa revisão.

Uso de álcool no Brasil e suas consequências negativas. De acordo com a Associação Pan-Americana de Saúde (Opas), o álcool é uma substância psicoativa com propriedades passíveis de causar dependência. Isso favorece a ocorrência de uma relação causal entre o uso nocivo do álcool e transtornos mentais e comportamentais, além da correlação de mais de duzentas doenças e lesões (Opas, 2019). Somada a esses dados, a compreensão do álcool como componente relevante para a socialização, sua disponibilidade facilitada e sua legalidade agravam seu uso indevido (Rosa \& Nascimento, 2015).

No Brasil, a população consumidora de álcool nos últimos 12 meses anteriores ao Levantamento Nacional de Álcool e Outras Drogas (Lenad) (Laranjeira et al., 2012) era de 62\% entre os homens e de $38 \%$ entre as mulheres. Corroborando com tal constatação, bom número de pesquisas relacionaram maior prevalência de casos de alcoolismo aos homens com alta vulnerabilidade social (Martins-Oliveira et al., 2016). Soma-se a esses dados o fato de que homens que consomem álcool apresentam uma maior proporção de anos perdidos em comparação a mulheres alcoolistas (Pérez-Pérez et al., 2016). Fato que vem ao encontro da preocupação associada ao contexto do presente estudo, pois os trabalhadores da construção civil são, em sua maioria, do sexo masculino.

Ademais, o alcoolismo tem implicações sobre a ocorrência de doenças crônicas sintomáticas e também moléstias assintomáticas, além de desencadear transtornos no âmbito afetivo, econômico e profissional do indivíduo (Kolasa, 2015; Martín-González et al., 2020). Na mesma direção, conforme ponderam Ritchie, Ritchie e Ward (2009), o alcoolismo acarreta consequências para os que estão em contato direto com os consumidores abusivos de bebidas alcóolicas. Esses acabam sofrendo com a ocorrência de violência doméstica, acidentes de trânsito, danos criminais e dificuldades fi- 
nanceiras decorrentes do alcoolismo.

Laranjeira, Pinsky, Zaleski, \& Caetano (2007) frisam que o consumo problemático de álcool está relacionado, especialmente, com as faixas etárias de até 44 anos. Dado que reforça a preocupação pública quanto ao número de afastamentos laborais por doenças relacionadas ao uso do álcool, dentre outras drogas. Estatística divulgada pela Previdência Social do Brasil (2013) indica que existiam 30,4 mil benefícios ativos no ano de 2013 em função de tal motivo.

Torna-se relevante salientar que as motivações para o consumo de álcool estão relacionadas a fatores intrínsecos e extrínsecos ao indivíduo. Dessa maneira, a situação familiar, o apoio social e as características pessoais dos indivíduos também estão associados ao abuso de álcool (Lopes, Marcon, \& Decesaro, 2015). Verifica-se, portanto, que, além de afetar o contexto pessoal com diversas consequências, o álcool interfere significativamente na conjuntura social do indivíduo, e nessa, o seu ambiente profissional.

Uso de álcool no contexto laboral. Marchand (2008) entende que a classe trabalhadora se encontra mais susceptível ao uso abusivo de substâncias deletérias, dentre estas o álcool obtém destaque. Ames, Grube e Moore (1997) evidenciaram que o consumo excessivo de álcool fora do trabalho, seja antes ou depois do expediente, considerando-se também a ressaca ocasionada, está diretamente relacionado a doenças do trabalho e conflitos com gestores. Tese que reforça a relevância de se abordar o tema tanto em âmbito acadêmico como laboral.

Sabe-se, ainda, que além das consequências para a saúde emocional do alcoolista, o consumo excessivo de álcool acarreta em problemas e incidentes no contexto laboral, tais como faltas repetitivas (Virtanen et al., 2018), acidentes (Zhang et al., 2020), incapacidades e diminuição da eficácia no trabalho (Gavioli et al., 2014). Ainda, no contexto do setor da construção civil, o álcool diminui as habilidades dos trabalhadores para executar tarefas básicas, como o alinhamento, ângulo e padrão de serviço (Dzeng et al., 2015).

Ademais, estudo que objetivou investigar as causas de acidentes de trabalho no contexto da construção civil, relevou que o comportamento inseguro dos trabalhadores é o motivo mais frequente e direto de acidentes (Zhang et al., 2020). Dessa forma, gerenciar e regular o comportamento dos trabalhadores deve reduzir muito os riscos nos canteiros de obras e a probabilidade de ocorrência de acidentes (Zhang et al., 2020) e isso inclui o posicionamento do gestor frente ao trabalhador alcoolista.

Ainda, argumenta-se que um canteiro de obras efetivamente gerenciado em seus incidentes terá maior probabilidade de identificar áreas problemáticas e, em consequência, ações preventivas poderão ser tomadas (Ayhan \& Tokdemir, 2019). Ratifica-se, assim, o papel fundamental do gestor frente aos acidentes e ao consumo de álcool no que concerne ao gerenciamento e monitoramento, conforme proposto neste estudo. Desse modo, a empresa e o gestor devem estar preparados para oferecer aos empregados alcoolistas iniciativas que lhe confiram saúde física, conforte psicológico e o fomento de relações saudáveis e labor seguro (Fernandes et al., 2015).

Diante dos aspectos mencionados, torna-se possível afirmar que o ambiente laboral tem importante papel no reconhecimento, intervenção e na adoção de práticas destinadas a melhorar a qualidade de vida do indivíduo alcoolista. Ações que podem contribuir efetivamente para a redução do consumo de álcool entre os trabalhadores (Bavafa et al., 2018). Conforme estudos relativos ao tema, evidencia-se que a falta de orientação em relação ao álcool e seus riscos contribui para o abuso de seu consumo (Fonseca, 2007; Mabuchi et al., 2007).

Em função disso, verifica-se a influência decisiva que os pro- gramas de prevenção de drogas, inclusive os referentes ao uso de bebidas alcoólicas, possuem sobre os trabalhadores (Bavafa et al., 2018; Brites et al., 2019). Nesse sentido, segundo o estudo de Sitzoglou et al. (2003), o trabalhador desintoxicado, na maior parte das situações, apresenta estabilidade emocional e profissional, bem como maior grau de estima, podendo retornar às suas atividades laborais e contribuir com a sociedade. Assim compreendido, passa-se a descrever a metodologia deste estudo.

\section{Método}

Para atender ao objetivo desta pesquisa, que consistiu em analisar a maneira como gestores atuam diante de contextos organizacionais da construção civil onde há colaboradores alcoolistas, delineou-se um estudo de natureza quantitativa, descritiva e de corte transversal.

\section{Participantes}

Considerando-se uma população de 774 empresas do ramo da construção civil cadastradas no município de Passo Fundo (RS), Brasil (Sebrae, 2020), o presente estudo caracterizou-se como amostral, contando com a participação de 50 dessas organizações. A técnica de amostragem adotada foi a não probabilística, por conveniência, pois selecionaram-se os elementos por estarem dispostos a participar da pesquisa (Malhotra, 2010). Como critério de inclusão, considerou-se as empresas que possuíam em seu quadro de pessoal no mínimo seis trabalhadores, excluindo-se aquelas constituídas como Microempreendedor Individual (MEI).

\section{Instrumentos}

Os dados primários foram perscrutados pela aplicação de um questionário, respondido por via eletrônica ou versão impressa pelos gestores das empresas $(n=50)$ de construção civil do município de Passo Fundo (RS). Antes de sua aplicação, o instrumento foi submetido à avaliação por um expert da área. Além disso, ocorreu um pré-teste com duas empresas do contexto em estudo, que obtiveram uma média de tempo de resposta de 7 minutos.

O instrumento de coleta de dados foi composto por três seções. A primeira buscou identificar o perfil da empresa e do gestor (dados sociodemográficos), enquanto a segunda seção compôs-se por perguntas relacionadas ao contexto de trabalho e a terceira a escala do tipo Likert de cinco pontos adaptada de Holanda (2013), 0 correspondendo a 'discordo totalmente' e 5 'concordo totalmente'. Tal escala abordou afirmativas sobre a situação da empresa e a visão do gestor sobre o alcoolismo no ambiente laboral e dividiu-se em duas grandes dimensões: i) questões referentes à situação da empresa no que se refere ao alcoolismo no ambiente laboral, composta pelas questões Q9/1 a Q9/13; e, ii) questões relativas à percepção do gestor sobre o alcoolismo e ambiente laboral, representadas pelo código Q9/14 a Q9/26.

\section{Procedimentos de Coleta de Dados e Cuidados Éticos}

Antes de se realizar a coleta dos dados, fez-se contato prévio com as empresas para garantir que estivessem situadas na cidade de Passo Fundo (RS) e fossem vinculadas ao ramo da construção civil (CNAE 4120-4), bem como que os gestores tivessem há seis meses ou mais executando serviços na empresa em cargos de liderança.

Atentando aos aspectos éticos, o projeto para este estudo foi submetido e aprovado pelo Comitê de Ética em Pesquisa (CEP) da IMED, parecer no 2.320.220, CAAE: 77335317.0.0000.5319, de acordo com as exigências contempladas nas Resoluções no 466 de 


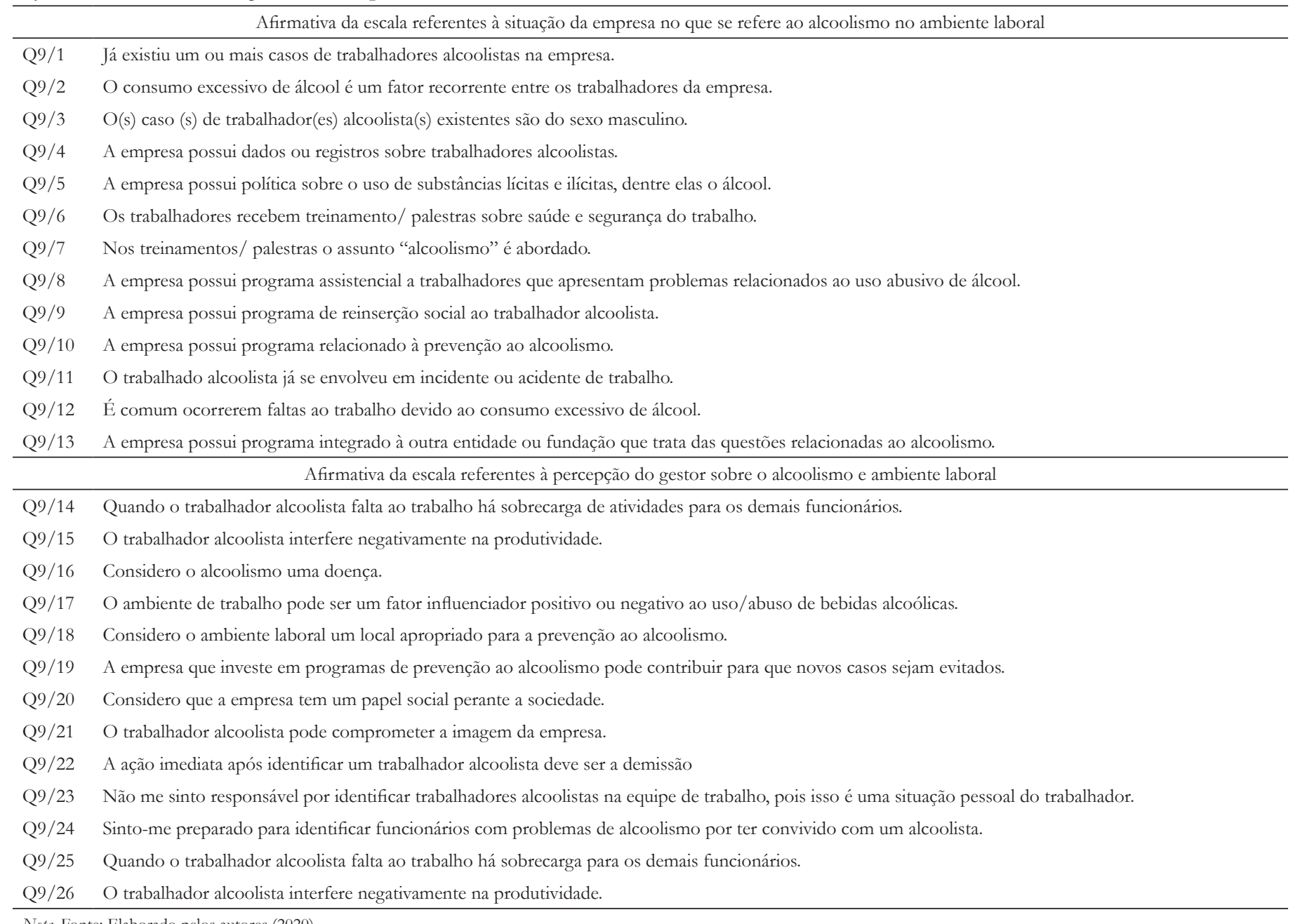

Nota. Fonte: Elaborado pelos autores (2020).

2012 e no 510/2016 do Conselho Nacional de Saúde (CNS), que dispõem sobre normas éticas para a realização de pesquisas com seres humanos. Todos os participantes tiveram acesso ao Termo de Consentimento Livre Esclarecido (TCLE), disponibilizado por meio eletrônico e impresso e, os que concordaram em participar, confirmaram terem sido informados dos objetivos da pesquisa $\mathrm{e}$ decidiram voluntariamente dela participar.

\section{Procedimento de Análise de Dados}

Os dados primários foram submetidos à análise estatística descritiva (média, frequência observada e relativa), realizada com o auxílio do software IBM SPSS Statistics [Statistical Package for the Social Sciences - SPSS (v. 26)]. Para tanto, analisou-se as seguintes variáveis: (i) situação da empresa em relação ao alcoolismo e ambiente laboral; e, (ii) a percepção do gestor sobre o alcoolismo e ambiente laboral. Após as análises descritivas, realizou-se o teste referente à normalidade dos dados obtidos utilizando-se o teste Kolmogorov-Smirnov [S-K], que acusou o desvio a normalidade dos dados $(p<0,005)$. A associação entre as variáveis qualitativas 'porte da empresa' e 'o consumo excessivo de álcool é um fator recorrente entre os trabalhadores da empresa', bem como as variáveis 'nível de formação dos gestores' e 'considero o alcoolismo uma doença' foi explorada por meio do teste de Qui-quadrado ( $\chi 2)$ para independência das variáveis. Ainda, considerou-se 'o alcoolismo uma doença' e a variável 'sexo', com o uso do teste U de Mann-Whitney.

Verificou-se, ainda, a confiabilidade da escala por meio do Alfa de Cronbach ( $\alpha$ ). Para a interpretação dos resultados, os pes- quisadores guiaram-se pelos parâmetros indicados por Streiner (2003), para quem valores abaixo de 0,7 - mas próximos a 0,6 - podem ser considerados como satisfatórios. Neste estudo o valor de $\alpha$ foi igual a 0,510 , considerado adequado em função do tamanho da $\operatorname{amostra}(n=50)$.

\section{Resultados e Discussão}

Compuseram a amostra desta pesquisa 50 empresas do setor da construção civil de empresas situadas na cidade de Passo Fundo (RS), Brasil. As características da amostra e dos respondentes são descritas na Tabela 2.

O tempo de atuação dos gestores pesquisados, em média, é de 7,9 anos $(D P=5,31)$ em cargos de diretores $(30 \%, n=15)$, gerentes $(26 \%, n=13)$, mestres de obras $(20 \%, n=10)$, técnicos em segurança do trabalho $(10 \%, n=5)$, administradores $(6 \%, \mathrm{n}=3)$, acionistas $(4 \%, n=2)$ e engenheiros do trabalho $(4 \%, n=1)$. A gestão é predominantemente realizada por indivíduos do sexo masculino, representado por $92 \%(n=46)$ da amostra. Fato corroborado pelo estudo de Purnell e Foster (2003), ao afirmarem que o trabalho é majoritariamente realizado por homens nos canteiros de obras e que a preocupação com as taxas de alcoolismo está associada em maior grau ao sexo masculino. Em relação ao nível de formação, 24 respondentes possuem ensino superior completo (48\%), enquanto 16 possuem ensino médio completo (32\%), os demais variaram de fundamental incompleto a outros níveis de formação $(20 \%)$.

As empresas participantes da pesquisa caracterizam-se, principalmente, pelo fato de terem mais de 20 empregados, ou seja, 
Tabela 2

Perfil dos gestores e das empresas da amostra

\begin{tabular}{|c|c|c|c|}
\hline \multicolumn{2}{|c|}{ Variavéis dos gestores } & Frequência & Porcentagem \\
\hline \multirow{2}{*}{ Sexo } & Masculino & 46 & $92 \%$ \\
\hline & Feminino & 4 & $8 \%$ \\
\hline \multirow{3}{*}{ Escolaridade } & Ensino fundamental & 4 & $8 \%$ \\
\hline & Ensino médio & 20 & $42 \%$ \\
\hline & Ensino superior & 24 & $50 \%$ \\
\hline \multirow{7}{*}{ Função } & Administrador & 3 & $6 \%$ \\
\hline & Diretor & 15 & $30 \%$ \\
\hline & Engenheiro & 2 & $4 \%$ \\
\hline & Gerente & 13 & $26 \%$ \\
\hline & Mestre de Obras & 10 & $20 \%$ \\
\hline & $\begin{array}{c}\text { Técnico em Segurança do } \\
\text { Trabalho }\end{array}$ & 5 & $10 \%$ \\
\hline & Sócio & 2 & $4 \%$ \\
\hline \multicolumn{2}{|c|}{ Variavéis das empresas } & Frequência & Porcentagem \\
\hline \multirow{3}{*}{$\begin{array}{l}\text { Número de } \\
\text { funcionários }\end{array}$} & Até 10 & 10 & $20 \%$ \\
\hline & de 11 a 20 & 14 & $28 \%$ \\
\hline & Mais de 20 & 26 & $52 \%$ \\
\hline \multirow{3}{*}{$\begin{array}{l}\text { Tempo da } \\
\text { empresa }\end{array}$} & Até 5 anos & 12 & $24 \%$ \\
\hline & De 6 a 10 anos & 21 & $42 \%$ \\
\hline & Acima de 10 anos & 17 & $34 \%$ \\
\hline \multirow{3}{*}{ Porte da empresa } & Microempresa & 13 & $26 \%$ \\
\hline & Pequena empresa & 26 & $52 \%$ \\
\hline & Média empresa & 11 & $22 \%$ \\
\hline
\end{tabular}

Nota. Fonte: Elaborado pelos autores (2020).

são de pequeno porte $(52 \%, n=26)$. Para essa classificação toma-se os conceitos de porte de empresas presente na Lei Complementar no 123/2006 (Brasil, 2006). Em relação ao tempo de atuação da empresa no ramo, 38 delas possuem acima de seis anos (76\%), com no mínimo um ano de atuação e no máximo 35 anos. Ainda, a média de tempo de atividade das empresas é de 11,54 $\operatorname{anos}(D P=8,37)$.

As afirmativas relacionadas à situação da empresa em relação ao alcoolismo e ambiente laboral na perspectiva do gestor, apresentaram concordância de $94 \%$ para casos de alcoolismo no ambiente laboral, com forte grau de concordância $(X=4,28 ; D P$ $=0,904)$. Por outro lado, $64 \%(n=32, D P=1,01)$ afirmam não ser o alcoolismo um fator recorrente entre os trabalhadores da empresa. Ainda, o teste de Qui-quadrado informa que o fato de o consumo excessivo de álcool ser um fator recorrente entre os trabalhadores não está associado com o porte da empresa $\left(\chi^{2}=\right.$ $2,360 ; g l=4 ; p>0,05)$.

Outro dado relevante é obtido na análise de que $96 \%$ ( $n=$ $48, D P=0,64)$ dos casos de alcoolismo ocorrem entre os homens, facilmente explicados por ser essa uma atividade ocupada predominantemente por trabalhadores do sexo masculino. Esses resultados corroboram com os achados de Gavioli et al. (2014), que verificaram uma maior frequência de casos de alcoolismo associada aos trabalhadores homens do setor da construção civil.

No que tange ao contexto laboral das organizações em estudo e seu posicionamento frente ao consumo de álcool dos trabalhadores, percebeu-se que essas apresentam um baixo índice de envolvimento com programas de prevenção. Isso, em função de que a maioria delas não possui política $(60 \%, n=30)$, programa assistencial $(92 \%, n=46)$, programa de reinserção do trabalhador alcoolista $(96 \%, n=48)$. Nem tampouco, programa preventivo $(90 \%, n=45)$ ou programa integrado à outra entidade $(94 \%, n=$ 47) relacionados ao alcoolismo ou uso de outras substâncias. Em complemento, Cheng e Cheng (2016) relataram que a prática de terceirização de mão de obra no setor dificulta a implementação de políticas sobre o uso de substância lícitas e ilícitas. Nesse sentido, tais elementos consolidam a necessidade de um olhar mais consistente de intervenção, relacionado a uma estrutura abrangente de bem-estar e prevenção ao consumo do álcool dentro das organizações (Bavafa et al., 2018).

Ademais, sabe-se que o registro de informações de incidentes ocorridos no canteiro de obras por parte do empregador é uma importante ferramenta para a adoção de ações preventivas (Ayhan \& Tokdemir, 2019). Contudo, em $86 \%(n=43, D P=1,03)$ das empresas da amostra não há dados ou registro sobre trabalhadores alcoolistas envolvidos em tais ocorrências, o que acarreta em dificuldades de monitoramento e a implementação de um modelo preditivo para casos de abuso de álcool.

Nas empresas em análise, $54 \%$ dos gestores afirmam que os trabalhadores recebem treinamentos ou palestras sobre saúde e segurança do trabalho, embora, 50\% dos pesquisados também concordem que o assunto alcoolismo não é abordado nessas atividades. No sentido dessas afirmações, entende-se que programas de segurança são a forma mais eficaz de reduzir acidentes e conscientizar os colaboradores, devendo, portanto, incluir abordagens em relação ao consumo de álcool (Gómez-Recasens et al., 2018).

O posicionamento e gerenciamento dos gestores frente aos acidentes de trabalho é essencial para assegurar o bem-estar físico dos trabalhadores (Zhang et al., 2020), o que significa o monitoramento de casos de alcoolismo no contexto laboral. Portanto, destaca-se que $62 \%(n=31 ; D P=1,01)$ dos gestores afirmam que $\mathrm{o}(\mathrm{s})$ trabalhador(res) alcoolista(s) não se envolveram em acidentes de trabalho. Por outro lado, as faltas ao trabalho representam $80 \%$ de grau de concordância na percepção dos entrevistados $(n=40$; $D P=0,93)$, característica também relatada no estudo de Virtanen et al. (2018).

No que se refere à percepção dos gestores, os dados analisados evidenciam que o grau de concordância entre os que consideram o alcoolismo uma doença é de $62,5 \%(n=30 ; D P=1,09)$. Embora esse número seja elevado, destaca-se que 20\% das respostas foram neutras, ou seja, não concordam nem discordam com a afirmativa. Ainda, 16,7\% discordam que o alcoolismo é uma doença. Para essas questões, o teste de Qui-quadrado informou que as respostas sobre considerar ou não o alcoolismo uma doença foi significativa, o que evidencia as diferenças de percepções de gestores, quando associadas ao nível de formação dos mesmos $\left(\chi^{2}=11,040 ; g l=4 ; p<0,05\right.$; Tabela 3). Ainda, essa associação foi de $33,9 \%$, pelo teste $\mathrm{V}$ de Cramer. Além disso, chama atenção os 11 e 18 gestores que consideram o alcoolismo uma doença e que possuem ensino médio e superior, respectivamente. $\mathrm{O}$ teste $T$ de Mann-Whitney informou que não há diferença quanto ao sexo dos gestores $(p>0,05)$ e as respostas em relação a afirmação 'considero o alcoolismo uma doença'.

Ainda considerando a opinião dos gestores frente ao alcoolismo, tem-se que $50 \%(n=25 ; D P=1,18)$ deles não se sen-

Tabela 3

Resultados do teste de Qui-quadrado da associação entre a formação dos gestores e considerar o alcoolismo uma doença

\begin{tabular}{cccc}
\hline Estatística & Valor & $g l$ & $\begin{array}{c}\text { Significância } \\
\text { Bilateral }\end{array}$ \\
\hline Qui-quadrado de Pearson & 11,040 & 4 & $0,026^{*}$ \\
Razão de verossimilhança & 13,948 & 4 & $0,007^{* *}$ \\
Associação linear por linear & 8,278 & 1 & $0,004^{* *}$ \\
$N$ de casos válidos & 48 & & \\
$V$ de Cramer $=0,339$ & & & \\
\hline
\end{tabular}

Nota. ${ }^{*} p<0,05,{ }^{* *} p<0,01$. Fonte: Elaborada pelos autores (2021). 
tem responsáveis por identificar trabalhadores alcoolistas na sua equipe, pois isso é uma situação pessoal do trabalhador. Contudo, cabe salientar a importância da atenção por parte dos gestores a possíveis casos de trabalhadores que consomem álcool na jornada de trabalho (Liang, Leung, \& Ahmed, 2021). Em dissonância com a afirmação anterior, $78 \%(n=39 ; D P=1,03)$ concordam fortemente $(X=3,90)$ que o trabalhador alcoolista pode comprometer a imagem da empresa.

Ademais, $56 \%(n=28 ; D P=1,16)$ discordam que a ação imediata após identificar o trabalhador alcoolista deve ser a demissão, o que reforça a importância do ambiente laboral no reconhecimento e intervenção do indivíduo alcoolista (Bavafa et al., 2018). Porém, embora os gestores entendam que ambiente laboral é um local apropriado para a prevenção $(54 \%, n=37)$ e $48 \%(n=24)$ deles concordem que esse contexto é um fator influenciador positivo ou negativo ao uso/abuso de bebidas alcóolicas, outros $36 \%$ $(n=18)$ discordam dessa afirmativa. Nesse sentido, tais dados divergem do estudo de Zhang et al. (2020), que entendem o papel primordial do gestor em relação à segurança dos trabalhadores do setor da construção civil.

Ainda, a maioria dos gestores concorda que a empresa tem um papel social perante à sociedade $(92 \%, n=46)$ e que investir em programas de prevenção pode contribuir positivamente para evitar novos casos $(82 \%, n=31)$. Essa visão corrobora com o estudo de Brites et al. (2019), que destacam a importância dos programas de prevenção para a redução de acidentes e para a qualidade de vida do trabalhador.

O consumo de álcool acarreta em consequências econômicas, sociais e também clínicas (Kolasa, 2015). O fato de sentir-se preparado para identificar um trabalhador alcoolista por já ter convivido com um deles apresentou 26\% $(n=13, D P=1,15)$ de concordância. Os dois principais sintomas clínicos que os gestores percebem em um trabalhador alcoolista são hálito alcóolico (29,6\%) e olhos lacrimejantes (19,8\%). Destaca-se, também, falta de coordenação motora e tremor fino nas exterminadas, ambas com 17,3\% cada (Figura 1).

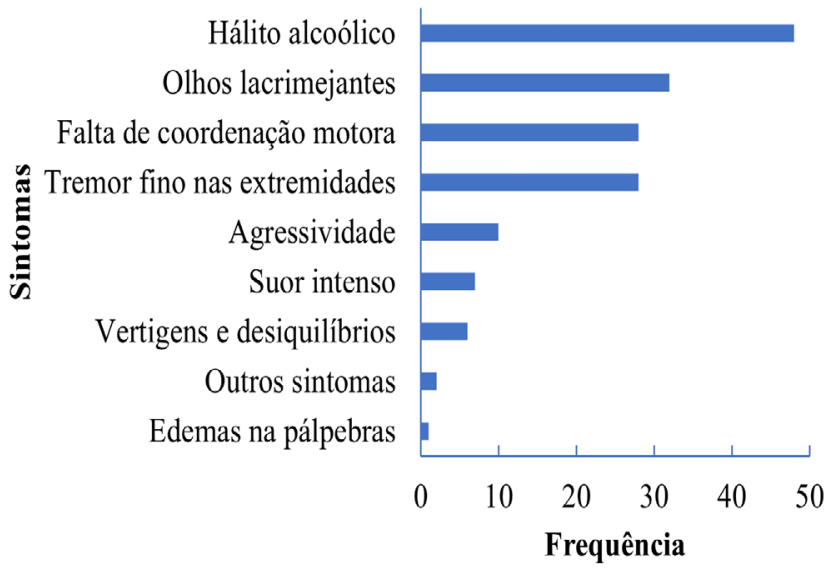

Figura 1. Sintomas mais comuns observados em trabalhadores alcoolistas, conforme de gestores participantes do estudo. Fonte: Elaborado pelos autores (2021).

Para a amostra em questão, as implicações relacionadas ao alcoolismo no ambiente de trabalho estão principalmente relacionadas a atrasos e baixa produtividade, ambos representando 19,8\% cada (Figura 2). Dados esses que reforçam os achados de Dzeng et al. (2015), quando afirmam que a qualidade do trabalho realizado é diretamente impactada pelo consumo de álcool, influenciando a capacidade de executar. Ainda, influenciando negativamente nas tarefas do canteiro de obras e podendo gerar acidentes de trabalho.

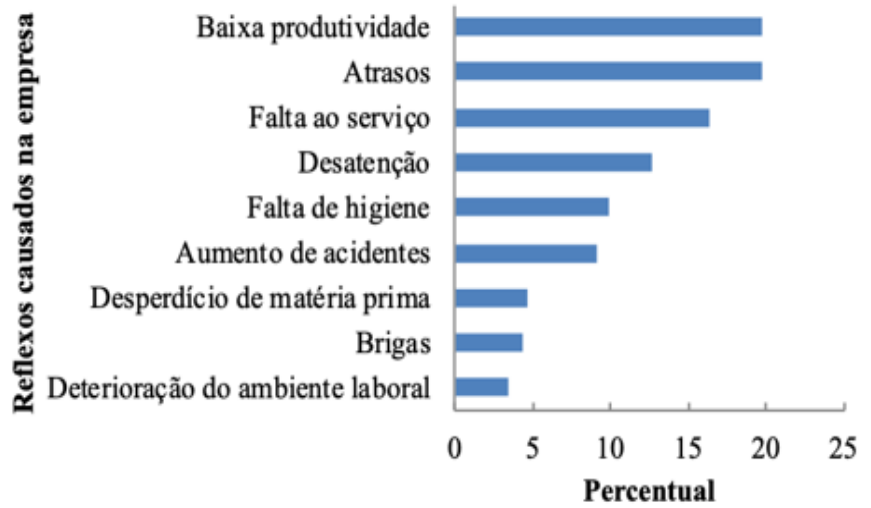

Figura 2. Reflexos que um trabalhador alcoolista causa na empresa, de acordo com a percepção dos gestores. Fonte: Elaborado pelos autores (2021).

$\mathrm{Na}$ análise sobre a perspectiva do gestor no que se refere à representação do trabalhador alcoolista para os demais colegas de trabalho (Figura 3), os resultados obtidos demostram que a maioria dos trabalhadores alcoolistas é tratado como os demais (56,7\%). Por outro lado, comentários que ferem a integridade representam $30 \%$. Ainda nesse contexto, ressalta-se o percentual de $10 \%$ para 'humilhação perante o grupo'. Destaca-se, portanto, a necessidade de atenção ao alcoolista, que pode estar travando lutas sociais e individuais (Fernandes et al., 2015) (Figura 3).

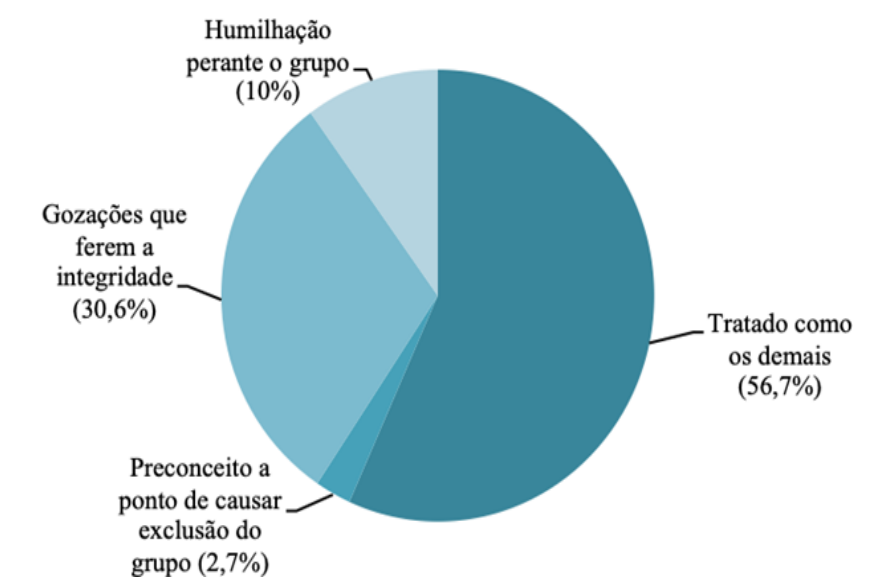

Figura 3. Representação do trabalhador alcoolista para os colegas de trabalho, na perspectiva do gestor. Fonte: Elaborado pelos autores (2021).

\section{Considerações Finais}

O alcoolismo é uma doença causadora de diversos danos aos indivíduos consumidores e apresenta uma série de reflexos no meio laboral. Assim, as empresas representam um papel importante para a sociedade, devendo estarem preparadas para atenderem as particularidades do seu ramo em relação a esse fenômeno. Tal condição deve ser considerada pois a sociedade vem exigindo atitudes éticas e responsáveis das organizações. Com efeito, ante ao exposto, os gestores assumem um papel fundamental nas ações de enfrentamento ao alcoolismo nas empresas.

Nesse sentido, este estudo teve o intuito de verificar de que maneira os gestores atuam diante de contextos organizacionais em que há colaboradores alcoolistas, a partir da identificação das consequências trazidas pelo alcoolismo à organização, tendo como foco o ramo da construção civil no município de Passo Fundo (RS), Brasil. O estudo demostrou que há casos de trabalhadores alcoolistas nas empresas participantes do estudo. Ao identificar as práticas da empresa no que se refere à assistência ao empregado alcoolista, os resultados apontaram que a maioria das empresas não possui programa assistencial, de reinserção ou registros relacionados ao trabalhador alcoolista. Ademais, as empresas não 
dispõem de programas preventivos próprios ou integrados à outra entidade.

No que diz respeito à percepção dos gestores, há diferença significativa de percepções sobre o alcoolismo ser considerado ou não uma doença quando associadas ao nível de formação dos mesmos. A maioria deles considera o alcoolismo uma doença, embora muitos tenham respostas neutras, o que pode significar uma incerteza sobre o assunto e contribuir para o número incipiente de iniciativas destinadas ao tema nas empresas. Além disso, os gestores concordam sobre o papel que essas têm para com a sociedade, bem como consideram o espaço laboral apropriado à prevenção, o que não se reflete na prática.

Os sintomas do trabalhador alcoolista mais percebidos pelos gestores foram o hálito alcóolico e os olhos lacrimejantes. Quanto aos reflexos mais prejudiciais à empresa, prevaleceram a baixa produtividade, atrasos e faltas ao serviço, confirmando, assim, que esses são efetivamente sentidos pela organização. Os gestores que participaram do estudo consideram que o trabalhador alcoolista pode comprometer a imagem da empresa, embora a demissão não seja apontada pela maioria como ação imediata. Esses fatores indicam que a empresa e os gestores têm consciência sobre o assunto, os reflexos e seus papéis frente ao problema. Porém, eles não possuem uma estrutura para trabalhar esses assuntos preventiva e corretivamente, o que é preocupante e limitante. Soma-se a isso o fato de se ter identificado que muitos trabalhadores alcoolistas são tratados pelos colegas com gozações, o que fere a integridade desses, podendo ser reflexo de uma desestrutura empresarial sobre o assunto.

Como contribuição, os resultados deste estudo oferecem outra percepção sobre a temática, visto que a maioria dos estudos voltados ao trabalhador alcoolista são feitos em área de saúde e tratam geralmente dos sintomas e consequências nocivas do alcoolismo para a saúde do indivíduo. Assim, buscou-se preencher as lacunas constatadas nesses estudos no que concerne, especificamente, à percepção de gestores sobre o trabalhador alcoolista e, ainda, quanto aos reflexos do alcoolismo na atividade laboral da construção civil.

Sob o ponto de vista gerencial, espera-se, a partir deste estudo, que os resultados obtidos forneçam informações capazes de auxiliarem na formulação de políticas que auxiliem o trabalhador alcoolista. Isso, tendo-se em vista que o alcoolismo se constitui em um dos problemas mais expressivos de saúde pública no Brasil e no mundo. Espera-se, também, que os achados deste estudo aportem informações de interesse para as entidades que atuam na prevenção, tratamento e ações de redução de danos causados pelo uso abusivo de álcool. Além disso, os resultados deste estudo, no contexto laboral de engenharia civil, podem também constituir-se em subsídios para empresas de outros setores, pois ainda são escassas informações sobre a temática, especialmente no que tange à percepção de gestores sobre o trabalhador alcoolista.

Por fim, aponta-se como limitação do estudo o tamanho da amostra. Ainda, a pesquisa poderia envolver gestores de diversos níveis organizacionais da empresa, bem como os trabalhadores e comparar as suas respostas, o que conjuntamente poderia servir de indicação para estudos futuros.

\section{Referências}

Ames, G. M., Grube, J. W., \& Moore, R. S. (1997). The relationship of drinking and hangovers to workplace problems: An empirical study. Journal of Studies on Alcohol, 58(1), 37-47. https://doi.org/10.15288/jsa.1997.58.37

Ayhan, B. U., \& Tokdemir, O. B. (2019). Predicting the outcome of construction incidents. Safety Science, 113, 91-104. https://doi.org/10.1016/i. ssci.2018.11.001
Bavafa, A., Mahdiyar, A., \& Marsono, A. K. (2018). Identifying and assessing the critical factors for effective implementation of safety programs in construction projects. Safety Science, 106, 47-56. https://doi.org/10.1016/i. ssci.2018.02.025

Bravo Ortiz, C. M., \& Marziale, M. H. P. (2010). El consumo de alcohol en personal administrativo y de servicios de una universidad del Ecuador. Revista Latino-Americana de Enfermagem, 18(spe), 487-495. https://doi. org/10.1590/S0104-11692010000700002

Brites, R. M. R., Abreu, A. M. M., \& Portela, L. F. (2019). Reduction of the Alcohol Consumption Among Workers Using a Brief Intervention. Texto \& Contexto - Enfermagem, 28. https://doi.org/10.1590/1980-265x$\underline{\text { tce-2018-0135 }}$

Calvo-Porral, C., \& Levy-Mangin, J.-P. (2019). Situational factors in alcoholic beverage consumption. British Food Journal, 121(9), 2086-2101. https://doi. org/10.1108/BFJ-01-2019-0002

Casarin, V. A., Lavarda, R., \& Silveira, J. S. T. Da. (2018). O alcoolismo no meio operário em uma empresa santiaguense de construção civil (RS). Intern. Journal of Profess. Bus. Review, 3(1), 111. https://doi.org/10.26668/ businessreview/2018.v3i1.93

Castro, K. C. de. (2009). O papel do local de trabalho no tratamento de trabalhadores alcoolistas. Revista Psicologia, 9(1), 108-119. Recuperado de http://pepsic.bvsalud.org/scielo.php?script $=$ sci arttext\&pid $=\mathrm{S} 1984$ $\underline{66572009000100007 \& \operatorname{lng}=\mathrm{pt \& nrm}=\mathrm{iso} \& \operatorname{lng}=\mathrm{pt}}$

Cheng, W.-J., \& Cheng, Y. (2016). Alcohol drinking behaviors and alcohol management policies under outsourcing work conditions: A qualitative study of construction workers in Taiwan. International Journal of Drug Policy, 28, 43-47. https://doi.org/10.1016/i.drugpo.2015.08.011

Cockell, F. F. (2008). Da enxada à colher de pedreiro: Trajetórias de vulnerabilidade social na construção civil (Tese de Doutorado), Universidade Federal de São Carlos, São Paulo, Brasil.

Dzeng, R.-J., Wang, S.-H., \& Fang, Y.-C. (2015). Quantitative evaluation of the impact of night shifts and alcohol consumption on construction tiling quality. Applied Ergonomics, 50, 226-236. https://doi.org/10.1016/i. apergo.2015.03.008

Fernandes, M. D. F. F. de F., Santos, F. S. M. dos, Santana, K. W. C. de, Teles, W. D. S., \& Silva, C. E. (2015). Consumo de álcool e sua influência no ambiente de trabalho da construção civil. Scire Salutis, 4(2), 28-46. https://doi. org/10.6008/spc2236-9600.2014.002.0004

Fonseca, F. F. da. (2007). Conhecimentos e opiniões dos trabalhadores sobre o uso e abuso de álcool. Escola Anna Nery, 11(4), 599-604. https://doi. org/10.1590/s1414-81452007000400007

Gavioli, A., Mathias, T. A. de F., Rossi, R. M., \& Oliveira, M. L. F. de. (2014). Risks related to drug use among male construction workers. Acta Paulista de Enfermagem, 27(5), 471-478. https://doi.org/10.1590/1982-0194201400077

Gómez-Recasens, M., Alfaro-Barrio, S., Tarro, L., Llauradó, E., \& Solà, R. (2018). A workplace intervention to reduce alcohol and drug consumption: a nonrandomized single-group study. BMC Public Health, 18(1), 1281. https:// doi.org/10.1186/s12889-018-6133-y

Holanda, M. A. M. (2013). Programas de apoio a usuários de álcool na construção civil: um estudo em empresas de Mossoró - RN. Universidade Federal Rural do SemiÁrido.

Junqueira, M. A. de B., Ferreira, M. C. de M., Soares, G. T., Brito, I. E. de, Pires, P. L. S., Santos, M. A. dos, \& Pillon, S. C. (2017). Uso de álcool e comportamento de saúde entre profissionais da enfermagem. Revista Da Escola de Enfermagem Da USP, 51. https://doi.org/10.1590/s1980220x2016046103265

Kolasa, K. (2015). The financial and health consequences of alcohol consumption - review of literature. Alcoholism and Drug Addiction, 28(3), 183-192. https://doi.org/10.1016/j.alkona.2015.10.001

Laranjeira, R., Pinsky, I., Zaleski, M., \& Caetano, R. (2007). I Levantamento nacional sobre os padrões de consumo de álcool na população brasileira. Em Secretaria Nacional de Políticas sobre drogas. Instituto Nacional de Ciência e Tecnologia para Políticas Públicas de Álcool e Outras Drogas (INPAD).

Laranjeira, R., Madruga, C. S., Pinsky, I., Caetano, R., Mirsuhiro, S. S., Castello, G. (2012). II Levantamento Nacional de Álcool e Outras Drogas. Instituto Nacional de Ciência e Tecnologia para Políticas Públicas de álcool e Outras Drogas (INPAD). São Paulo: UNIFESP.

Lette, A., Ambelu, A., Getahun, T., \& Mekonen, S. (2018). A survey of workrelated injuries among building construction workers in southwestern Ethiopia. International Journal of Industrial Ergonomics, 68, 57-64. https://doi. org/10.1016/j.ergon.2018.06.010

Liang, Q., Leung, M., \& Ahmed, K. (2021). How adoption of coping behaviors determines construction workers' safety: A quantitative and qualitative investigation. Safety Science, 133, 105035. https://doi.org/10.1016/j. ssci.2020.105035

Lima, A. I. O., Dimenstein, M., Figueiró, R., Leite, J., \& Dantas, C. (2019). Prevalência de Transtornos Mentais Comuns e Uso de Álcool e Drogas entre Agentes Penitenciários. Psicologia: Teoria e Pesquisa, 35. https://doi. org/10.1590/0102.3772e3555 
Lopes, A. P. A. T., Marcon, S. S., \& Decesaro, M. das N. (2015). Abuso de bebida alcoólica e sua relação no contexto familiar. Estudos de Psicologia, 20(1), 21-30. https://doi.org/10.5935/1678-4669.20150004

Mabuchi, A. dos S., Oliveira, D. F. de, Lima, M. P. de, Conceição, M. B. da, $\&$ Fernandes, H. (2007). The use of alcohol by workers of the garbage collection service. Revista Latino-Americana de Enfermagem, 15(3), 446-452. https://doi.org/10.1590/S0104-11692007000300013

Malhotra, N. (2010). Pesquisa de Marketing: foco na decisão (3rd ed.). Pearson.

Marchand, A. (2008). Alcohol use and misuse: What are the contributions of occupation and work organization conditions? BMC Public Health, 8(1), 333. https://doi.org/10.1186/1471-2458-8-333

Martín-González, C., Ribot-Hernández, I., Fernández-Rodríguez, C., GonzálezNavarrete, L., Vera-Delgado, V., Rodríguez-Gaspar, M., Martínez-Riera, A., \& González-Reimers, E. (2020). Incidence of cancer and mortality among heavy alcoholics. Clinical Nutrition ESPEN. https://doi.org/10.1016/i. clnesp.2020.02.012

Martins-Oliveira, J. G., Jorge, K. O., Ferreira, R. C., Ferreira, E. F. e, Vale, M. P., \& Zarzar, P. M. (2016). Risk of alcohol dependence: prevalence, related problems and socioeconomic factors. Ciência \& Saúde Coletiva, 21(1), 17-26. https://doi.org/10.1590/1413-81232015211.00652015

Mendonça, A. K. R. H., Jesus, C. V. F. de, \& Lima, S. O. (2018). Fatores Associados ao Consumo Alcoólico de Risco entre Universitários da Área da Saúde. Revista Brasileira de Educação Médica, 42(1), 207-215. https://doi. org/10.1590/1981-52712018v42n1rb20170096

Nascimento, E. C. do, Nascimento, E., \& Silva, J. de P. (2007). Uso de álcool e anfetaminas entre caminhoneiros de estrada. Revista de Saúde Pública, 41(2), 290-293. https://doi.org/10.1590/S0034-89102007000200017

Organização Pan-Americana de Saúde (2019). Folha Informativa: Álcool. Recuperado de https://www.paho.org/pt/node/4825

Pérez-Pérez, E., Cruz-López, L., Hernández-Llanes, N. F., Gallegos-Cari, A., Camacho-Solís, R. E., \& Mendoza-Meléndez, M. Á. (2016). Años de Vida Perdidos (AVP) atribuibles al consumo de alcohol en la ciudad de México. Ciência \& Saúde Coletiva, 21(1), 37-44. https://doi.org/10.1590/1413$\underline{81232015211.09472015}$

Previdência Social do Brasil (2013). Previdência em Questão: cresce o número de afastamentos por doenças relacionadas ao uso de álcool. Recuperado de http:// sa.previdencia.gov.br/site/arquivos/office/28 130909-141429-414.pdf

Purnell, L., \& Foster, J. (2003). Cultural aspects of alcohol use. Drugs and Alcohol Today, 3(2), 3-8. https://doi.org/10.1108/17459265200300012

Ritchie, C., Ritchie, F., \& Ward, R. (2009). A good night out: alcohol-related behaviours in young adults. Worldwide Hospitality and Tourism Themes, 1(2), 169-193. https://doi.org/10.1108/17554210910962549

Rosa, L. F. de A., \& Nascimento, A. R. A. (2015). Representações sociais de bebida alcoólica para homens universitários. Arquivos Brasileiros de Psicologia, 67(1), 3-19. Recuperado de http://pepsic.bvsalud.org/scielo. php? script $=$ sci arttext\&pid $=$ S1809-52672015000100002

Sebrae. (2020). Perfil das cidades gaúchas: Passo Fundo. Recuperado de https:// datasebrae.com.br/municipios/rs/Perfil Cidades Gauchas-Passo Fundo. pdf

Sitzoglou, K., Papadopoulos, K., Tsiptsios, J., Charalampous, C., Androutsos, A., \& Adamopoulou, M. (2003). Impact of alcohol use and its related disorders at work in relavance with family history of alcoholism. Annals of General Hospital Psychiatry, 1, 21.

Soares, J. F. de S., Cezar-Vaz, M. R., Cardoso, L. da S., Soares, M. C. F., Costa, V. Z. da, \& Almeida, M. C. V. de. (2007). O risco do uso de drogas no trabalho portuário: estudo no extremo sul do Brasil. Escola Anna Nery, 11(4), 593-598. https://doi.org/10.1590/S1414-81452007000400006

Streiner, D. L. (2003). Being Inconsistent About Consistency: When Coefficient Alpha Does and Doesn't Matter. Journal of Personality Assessment, 80(3), 217-222.

Virtanen, M., Ervasti, J., Head, J., Oksanen, T., Salo, P., Pentti, . . \& \& Kivimäki, M. (2018). Lifestyle factors and risk of sickness absence from work: a multicohort study. The Lancet Public Health, 3(11), e545-e554. https://doi. org/10.1016/S2468-2667(18)30201-9

World Health Organization (2018). Global status report on alcohol and health. Recuperado de https://www.who.int/publications/i/item/9789241565639

Zhang, W., Zhu, S., Zhang, X., \& Zhao, T. (2020). Identification of critical causes of construction accidents in China using a model based on system thinking and case analysis. Safety Science, 121, 606-618. https://doi.org/10.1016/i. $\underline{\text { ssci.2019.04.038 }}$
Informações sobre os autores:

Jaqueline Garcia Fisch

Rua Senador Pinheiro, 304

Passo Fundo, RS, Brasil

E-mail: jaqueline.fisch@hotmail.com

\section{Tainara Kaspary}

E-mail: tainarakaspary@gmail.com

Cintia Sonale Rebonatto

E-mail: cintiasonale@gmail.com

\section{Carlos Costa}

Rua Senador Pinheiro, 304

Passo Fundo, RS, Brasil

E-mail: carlos.costa1@gmail.com 